\title{
INDIVIDUAL POSITIONING: A COMPARATIVE STUDY OF ADJUVANT BREAST RADIOTHERAPY IN THE PRONE VERSUS SUPINE POSITION
}

\author{
Zoltán Varga, Katalin Hideghéty, M.D., Ph.D., Tamás Mezö, Alíz Nikolényi, M.D., \\ László Thurzó, M.D., Ph.D., and Zsuzsanna Kahán, M.D., Ph.D. \\ Department of Oncotherapy, University of Szeged, Szeged, Hungary
}

\begin{abstract}
Purpose: To study breast radiotherapy in the prone vs. supine positions through dosimetry and clinical implementation.

Methods and Materials: Conformal radiotherapy plans in 61 patients requiring only breast irradiation were developed for both the prone and supine positions. After evaluation of the of the first 20 plan pairs, the patients were irradiated in the prone or supine position in a randomized fashion. These cases were analyzed for repositioning accuracy and skin reactions related to treatment position and patient characteristics.

Results: The planning target volume covered with 47.5-53.5 Gy in the prone vs. the supine position was $85.1 \% \pm$ $4.2 \%$ vs. $89.2 \pm 2.2 \%$, respectively $(p<0.0001)$. Radiation exposure of the ipsilateral lung, expressed in terms of the mean lung dose and the $V_{20 \mathrm{G} y}$, was dramatically lower in the prone vs. supine position $(p<0.0001)$, but the doses to the heart did not differ. There was no difference in the need to correct positioning during radiotherapy, but the extent of displacement was significantly higher in the prone vs. supine position $(p=0.021)$. The repositioning accuracy in the prone position exhibited an improvement over time and did not depend on any patient-related parameters. Significantly more radiodermatitis of Grade 1-2 developed following prone vs. supine irradiation $(p=0.025)$.

Conclusions: Conformal breast radiotherapy is feasible in the prone position. Its primary advantage is the substantially lower radiation dose to the ipsilateral lung. The higher dose inhomogeneity and increased rate of Grade 1-2 skin toxicity, however, may be of concern. (c) 2009 Elsevier Inc.
\end{abstract}

Breast cancer, Conformal radiotherapy, Prone treatment position, Supine treatment position, Repositioning accuracy.

\section{INTRODUCTION}

Postoperative radiotherapy has become an integral part of the complex treatment of breast cancer. The risk of late radiogenic sequelae such as lung fibrosis, cardiovascular events, or secondary cancers increases with radiation exposure of the organs at risk (OARs) (1-4), and selective irradiation of the target organ is therefore mandatory. The simplest way to protect the OARs during breast radiotherapy is individual patient positioning. It has been observed that a prone position during breast radiotherapy results in a substantially lower dose to OARs such as the ipsilateral lung (5-9) and the heart $(5,8)$, with the additional advantage of improved dose homogeneity $(5,6,9)$. This mode of positioning has been shown to be feasible $(10,11)$, even in obese patients $(8)$, and to provide a similar long-term outcome and toxicity as with standard supine tangents $(11,12)$. Because we had earlier found the prone position to be helpful in a few difficult cases, we set out to perform a prospective study to compare radiotherapy in the prone position with our usual technique in the supine position with excellent repositioning accuracy. The study comprised two phases: the first phase served as a setup period for the acquisition of experience with patient positioning and radiotherapy planning in the prone position, but the radiotherapy was in fact delivered in the conventional supine position; in the second phase, radiotherapy administered in the prone vs. the supine position in a randomized fashion was studied. The radiotherapy plans were analyzed for the overall study population, whereas the implementation of breast radiotherapy in the prone position was the subject of only the second phase of the study. We aimed to identify those patients who benefit most from prone positioning by means of dosimetry (dose homogeneity and
Reprint requests to: Zsuzsanna Kahán, Department of Oncotherapy, University of Szeged, Korányi fasor 12, H-6720 Szeged, Hungary. Tel: (+36) 62-545406; Fax: (+36) 62-545922; E-mail: kahan@onko.szote.u-szeged.hu

A portion of this article was presented at the 27th Annual Meeting of the European Society of Therapeutic Radiology and Oncology, Göteborg, Sweden, September 14-18, 2008.
Conflict of interest: Katalin Hideghéty plays an advisory role in the technical development at ORFIT, for which she receives regular honoraria.

Acknowledgments - The authors are grateful to Dr. Krisztina Boda for statistical analysis counseling.

Received Sept 3, 2008, and in revised form Oct 24, 2008. Accepted for publication Oct 28, 2008. 
protection of the OARs) and feasibility (including repositioning accuracy).

\section{METHODS AND MATERIALS}

The study was approved by the Institutional Review Board of the University of Szeged, and all enrolled patients gave their written informed consent before being registered in the study.

Early breast cancer patients after surgery requiring only radiotherapy of the operated breast were included in the study. No restriction existed regarding the size of the breast or the patient.

In the first phase of the study $(n=20)$, although radiotherapy planning was performed in both positions, all patients received radiotherapy in the supine position. The 41 patients enrolled in the second phase were randomized to radiotherapy in the prone vs. the supine position, but the position for radiotherapy randomized to the patient was blinded to the physician who performed the contouring.

The patients were positioned on the supine thorax and the prone breast modules of the AIO (All In One) Solution (ORFIT, Wijnegem, Belgium) system, which contains special cushion sets fixed to a universal baseplate. In the supine position, the patient was laid on a $15^{\circ}$ thorax wedge cushion with both arms elevated, resting on an arm support, and held on an adjustable grip pole. The head was placed in the head support secured to a supplementary baseplate attached to the thorax cushion. In the prone position, the head was resting on a pillow, both arms were placed superolaterally, supported by the cranial part of the prone breast cushion, and the target breast lay across the semicircular aperture of the platform. The patient was rotated slightly to allow the ipsilateral chest wall to extend into the aperture. A thermoplastic mask (five-point fixation, breast precut; ORFIT) was applied in the supine position, molded around the chin, the neck, the thorax (excluding the target breast), and the abdomen. The opposite breast was covered with the mask and carefully positioned away from the radiation fields. Mask fixation was not used in the prone position, but a polyfoam wedge was placed under the contralateral breast to displace it. On the basis of experience gained during the first phase of the study, in the second 41 patients, a different polyfoam wedge was applied as a new development of the AIO system for better protection of the opposite breast (Fig. 1). Positioning landmarks were drawn on the skin or the mask, using two lateral lasers and one overhead laser. All patients were scanned on a Somatom Emotion 6 CT simulator (Siemens, Erlangen, Germany) in both positions. The planning target volume (PTV) and OARs were contoured on the CT slices throughout the entire planning volume in the XiO (CMS, Maryland Heights, MO) treatment planning system, according to the local protocol (13). The PTV was defined as the entire breast delineated on the CT data set, extending to within $4 \mathrm{~mm}$ of the skin surface. Treatment plans were developed by applying conventional 6-MV tangential photon fields set up isocentrically and a median of 2 (range, 1-3) individually weighted 6/15-MV segmental fields superimposed on the tangential fields by using a multileaf collimator. Wedges were used in almost all cases. A mean dose to the PTV of $50 \mathrm{~Gy}$ and a uniform distribution $( \pm 10 \%)$ of the prescribed dose to $95 \%$ of the PTV were aimed for. Dose homogeneity within the PTV was characterized by the volume of the breast receiving at least $47.5 \mathrm{~Gy}$ but less than 53.5 $\mathrm{Gy}\left(\mathrm{V}_{95 \%-107 \%}\right)$. Radiation exposure of the OARs (the volume of the ipsilateral lung receiving more than $20 \mathrm{~Gy}\left[\mathrm{~V}_{20 \mathrm{~Gy}}\right]$, the mean lung dose [MLD], the mean dose to the heart [MHD], the volume of the heart receiving more than 25 or $30 \mathrm{~Gy}\left[\mathrm{~V}_{25 \mathrm{~Gy}}\right.$ and $\left.\mathrm{V}_{30 \mathrm{~Gy}}\right]$, the volume of the contralateral breast receiving more than 5 Gy $\left[\mathrm{V}_{5 \mathrm{~Gy}}\right]$, and the mean dose to the contralateral breast) was registered
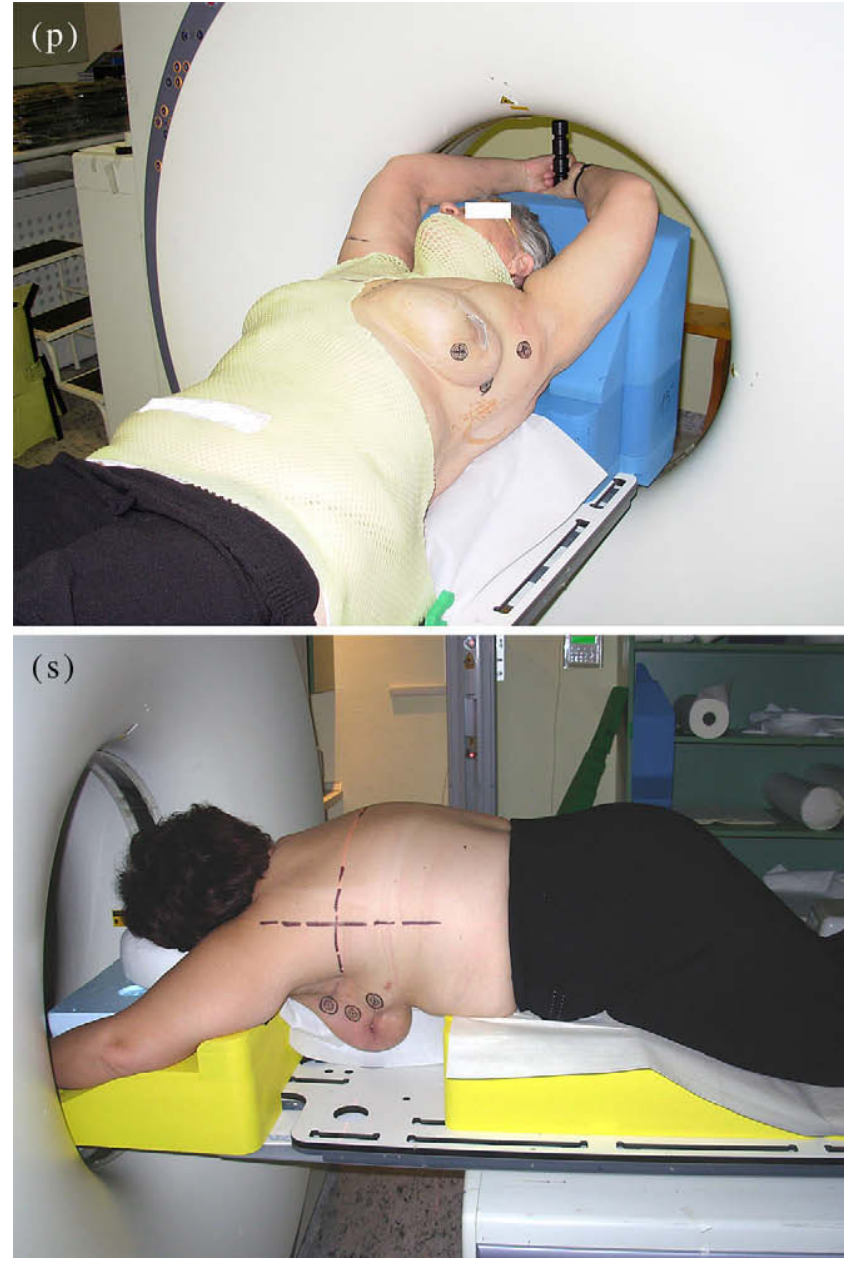

Fig. 1. Typical supine and prone positioning during breast radiotherapy.

in both positions. The central lung distance (CLD) and breast separation were determined in the supine position as measures of the patient anatomy.

The objectives in the second phase of the study were patient adherence to the protocol, repositioning accuracy, and toxicity during radiotherapy. Before the commencement of radiotherapy, the position of the isocenter in the patient was checked under the CT simulator. The necessary displacement in three dimensions was registered as the first datum of the repositioning accuracy. Radiotherapy was delivered with a linear accelerator (Primus, Siemens) in five fractions per week. The accuracy of patient repositioning during radiotherapy was checked three times per week with an electronic portal imaging device (Beamview version 2.2, Siemens), with the help of radio-opaque markers placed on the skin and mask as reference markers. (The dose delivered by portal imaging was taken into consideration in the calculation of the final dose received by the patient.) One portal image for one of the tangentional beams was recorded and compared with the corresponding beam's eye view digitally reconstructed radiograph generated from the planning system. The need to correct the position of the table in two dimensions was established and recorded by one or two physicians (AN or ZK). Analysis of each port image involved determination of the distances between the radio-opaque skin markers, and measurements of the CLD, the lung area included in the field, the central flash distance, and the inferior central margin $(14,15)$. The action level was set at $3 \mathrm{~mm}$. Systematic and random errors generated from the three-dimensional 
vector of displacement during the CT simulation and the two dimensional vector of displacement during the radiotherapy were calculated according to conventional definitions $(16,17)$. Acute skin reactions (graded by the Common Terminology Criteria for Adverse Events, version 3.0) were compared in 41 patients randomized to radiotherapy in the prone vs. supine position, at the end of the whole breast irradiation. The relations between the data obtained by analysis of the radiotherapy plans and repositioning accuracy vs. the patient characteristics were analyzed with the Student $t$ test, the chi-square test, regression analysis, analysis of variance, and logistic regression. Statistical analysis was performed with SPSS 11.0 for Windows.

\section{RESULTS}

The first phase of the study and the second feasibility phase involved 20 and 41 patients, respectively. The mean ( \pm SD) age of the overall study population was $56.0 \pm 9.6$ (range, 29.3-73.9), and that of the second phase was $56.6 \pm 9.9$ (range, 29.3-73.6) years. Twenty-seven patients underwent right-sided and 34 left-sided breast irradiation. The age, weight, waist, hip size, and breast separation did not differ significantly between patients randomized to radiotherapy in the prone or the supine position (Table 1). Tumor bed boost irradiation and systemic treatments did not differ significantly between the two groups.

\section{Radiotherapy plans for the prone vs. the supine position}

The radiotherapy plans were first analyzed in the overall population. Mean ( \pm SD) percentage PTV covered by 47.5-53.5 Gy $\left(\mathrm{V}_{95 \%-107 \%}\right)$ in the prone vs. the supine position was $85.1 \pm 4.2 \%$ and $89.2 \pm 2.2 \%$, respectively $(p<0.0001)$. Dose homogeneity did not depend on PTV or breast separation. The irradiated volume of and the dose to the ipsilateral lung, determined in terms of MLD and $\mathrm{V}_{20 \mathrm{~Gy}}$, were dramatically lower in the prone position than in the supine position (Table 2). No significant difference was detected in the mean dose to the heart and the volumes of the heart receiving at least $25 \mathrm{~Gy}$ or $30 \mathrm{~Gy}$ in 34 left-sided breast cancer patients according to their position during radiotherapy (Table 2). The first 20 pairs of treatment plans revealed significantly higher doses to the contralateral breast in the prone position than in the supine position. In the second phase of the study $(n=41)$, as a consequence of the more complete displacement of the opposite breast due to the use of a new polyfoam wedge, there was no longer any significant difference (Table 3 ).

We hoped to identify parameters related to patient anatomy that indicate high lung doses if radiotherapy is given in the supine position to select those patients who would benefit most from radiotherapy in the prone position. With regard to the volume of the target breast, breast separation, and CLD, only CLD was significantly associated with MLD $(r=0.843, p<0.0001)$ and $\mathrm{V}_{20 \mathrm{~Gy}}(r=0.733, p<0.0001)$.

\section{Implementation of breast radiotherapy in the prone position}

In the second phase of the study, adherence to the study protocol, repositioning accuracy, and early skin reactions 
Table 2. Radiation doses to the ipsilateral lung and the heart in the overall study population (mean \pm SD)

\begin{tabular}{llcrrr}
\hline & \multicolumn{2}{c}{ Lung $(n=61)$} & & \multicolumn{2}{c}{ Heart $(n=34)$} \\
\cline { 2 - 3 } & MLD $(\mathrm{Gy})$ & $\mathrm{V}_{\text {20Gy }}(\%)$ & & Mean dose $(\mathrm{Gy})$ & $\mathrm{V}_{25 \mathrm{~Gy}}(\%)$ \\
\hline Supine & $7.45 \pm 2.62$ & $14.3 \pm 5.4$ & & $3.51 \pm 2.33$ & $\mathrm{~V}_{30 \mathrm{~Gy}}(\%)$ \\
Prone & $2.02 \pm 1.23$ & $3.3 \pm 2.5$ & & $3.18 \pm 1.31$ & $4.7 \pm 4.6$ \\
$p$ value & $<0.0001$ & $<0.0001$ & 0.413 & $3.6 \pm 2.5$ & 0.171 \\
\hline
\end{tabular}

Abbreviation: MLD = mean lung dose.

were analyzed. The protocol was tolerated well by all patients; only one treated in the prone position required a 1-week break because of radiodermatitis. It was necessary to correct the location of the isocenter in the simulator or the position of the table during radiotherapy in $20.3 \%$ (61/ $301)$ and $20.3 \%(62 / 306)$ of all checks in the prone and the supine position, respectively $(p=0.999)$. The mean length of the displacement vector was $8.06 \pm 4.66 \mathrm{~mm}$ (range, $3.00-22.56 \mathrm{~mm}$ ) and $6.60 \pm 3.05 \mathrm{~mm}$ (range, 3.00-21.19 $\mathrm{mm})$ in the prone and supine positions, respectively ( $p=$ 0.021 ). The population random errors were $3.89 \mathrm{~mm}$ and $2.97 \mathrm{~mm}$, whereas the population systematic errors were $0.86 \mathrm{~mm}$ and $0.82 \mathrm{~mm}$ for the prone and the supine position, respectively. The random errors in the two groups are shown in Table 4. A trend was detected for better overall repositioning accuracy in the supine position $(p=0.061)$. We analyzed whether repositioning accuracy changed from patient to patient during the study period. The individual random errors for repositioning in the prone position decreased with time, whereas no change was detected in the group randomized to radiotherapy in the supine position (Fig. 2). Repositioning accuracy in the prone position did not depend on any patientrelated parameter. In the supine position, however, it was significantly related to lower weight $(p=0.01)$, body mass index $(p=0.011)$, waist size $(p=0.039)$, volume of the ipsilateral breast $(p=0.007)$, and breast separation $(p=0.001)$. Grade 1 radiodermatitis developed in $55 \%$ and $38.1 \%$ of patients and Grade 2 radiodermatitis in $35 \%$ and $19.5 \%$ of the patients receiving radiotherapy in the prone or the supine position, respectively $(p=0.025)$. Acute skin reactions were not related to dose homogeneity in the PTV or the random errors for repositioning, regarded as measures of systematic and random overdosage, respectively.

\section{DISCUSSION}

We evaluated our initial experience regarding the dosimetry and feasibility of conformal breast radiotherapy in the prone position and identified its place in everyday practice. Our results indicate that its primary advantage is the significantly reduced radiation exposure of the ipsilateral lung. The doses to the heart and the contralateral breast are similar in the prone and supine positions. Special practice in and attention to accurate repositioning are necessary if the prone position is applied, and dose inhomogeneity and acute skin reactions may increase slightly.

There have been few studies on prone breast radiotherapy. Some focused on dose distribution $(6,7,9,18)$ and others on clinical implementation $(11,12,14,19,20)$; only one study with both dosimetric aspects and feasibility (10). This study is the first randomized clinical trial to compare breast radiotherapy in the prone vs. the supine position.

Use of the prone position during breast radiotherapy raises special considerations because of the altered shape, motion, and position of the organs present in the region. The altered shape of the target breast hanging down across the aperture of the positioning device results in a different dose distribution relative to that in the supine position. Improved dose uniformity, particularly avoidance of an overdosage within the PTV, have been associated with a better cosmetic outcome $(21,22)$. A higher dose inhomogeneity is related to larger breasts if conventional tangent beams are used (21). Buijsen et al. (9) compared prone and supine breast irradiation in 10 patients with pendulous breasts, and concluded that the dose homogeneity was better in the prone than the supine position. In fact, this was based on a comparison of the PTV overdosed $\left(\mathrm{V}_{105 \%}\right.$ and $\left.\mathrm{V}_{107 \%}\right)$ in the supine vs. prone position, but the significantly lower mean dose and PTV coverage representing an underdosage were neglected. Similarly, in another study (6), larger volumes receiving $>52.5$ Gy within the PTV were found in the supine than the prone position, but no other information on dose distribution was reported. We examined $\mathrm{V}_{95 \%-107 \%}$ as a measure of dose homogeneity within the PTV, according to International Commission on Radiation Units and Measurements Report 62 (23), and found that the dose distribution was significantly more

Table 3. Radiation dose to the opposite breast in the two consecutive cohorts of the study

\begin{tabular}{|c|c|c|c|c|c|c|}
\hline & \multicolumn{2}{|c|}{ First phase $(n=20)$} & \multicolumn{2}{|c|}{ Second phase $(n=41)$} & \multicolumn{2}{|c|}{$p$ for first vs. second phase } \\
\hline Prone & $1.26 \pm 0.78$ & $4.5 \pm 3.4$ & $0.74 \pm 0.44$ & $2.2 \pm 2.0$ & 0.00092 & 0.001 \\
\hline$p$ for supine vs. prone & 0.0038 & 0.0057 & 0.162 & 0.159 & & \\
\hline
\end{tabular}


Table 4. Random errors for repositioning in the prone and supine positions

\begin{tabular}{lcc}
\hline & Mean \pm SE $(\mathrm{mm})$ & Median $(\mathrm{mm})$ \\
\hline Supine & $2.75 \pm 0.27$ & 2.58 \\
Prone & $3.46 \pm 0.37$ & 3.48 \\
$p$ value & 0.061 & \\
\hline
\end{tabular}

uniform in the supine position, regardless of the size or shape of the target breast. None of the radiotherapy plans indicated measurable volumes receiving $>53.5 \mathrm{~Gy}$. Our dose prescription strategy was different from those of Buijsen et al. (9), and Griem et al. (6). A mean dose of $50 \mathrm{~Gy}$ was prescribed to the entire PTV, provided that the dose range is between 45 and 55 Gy in at least $95 \%$ of the PTV, instead of specifying a dose to a dose prescription point. We believe that our approach reliably represents the dose homogeneity within the PTV. Goodman et al. (18) reported a simplified intensity-modulated radiation therapy (IMRT) technique that improved dose homogeneity within the target breast in the prone position compared with the unacceptably high doses generated if conventional tangents were used. The greatest improvement was seen in women with the most pendulous breasts. Although in our study dose uniformity was acceptable in both positions, in certain cases, the IMRT approach could be followed to prevent the early and late consequences of dose inhomogeneity. In accordance with these data, in an
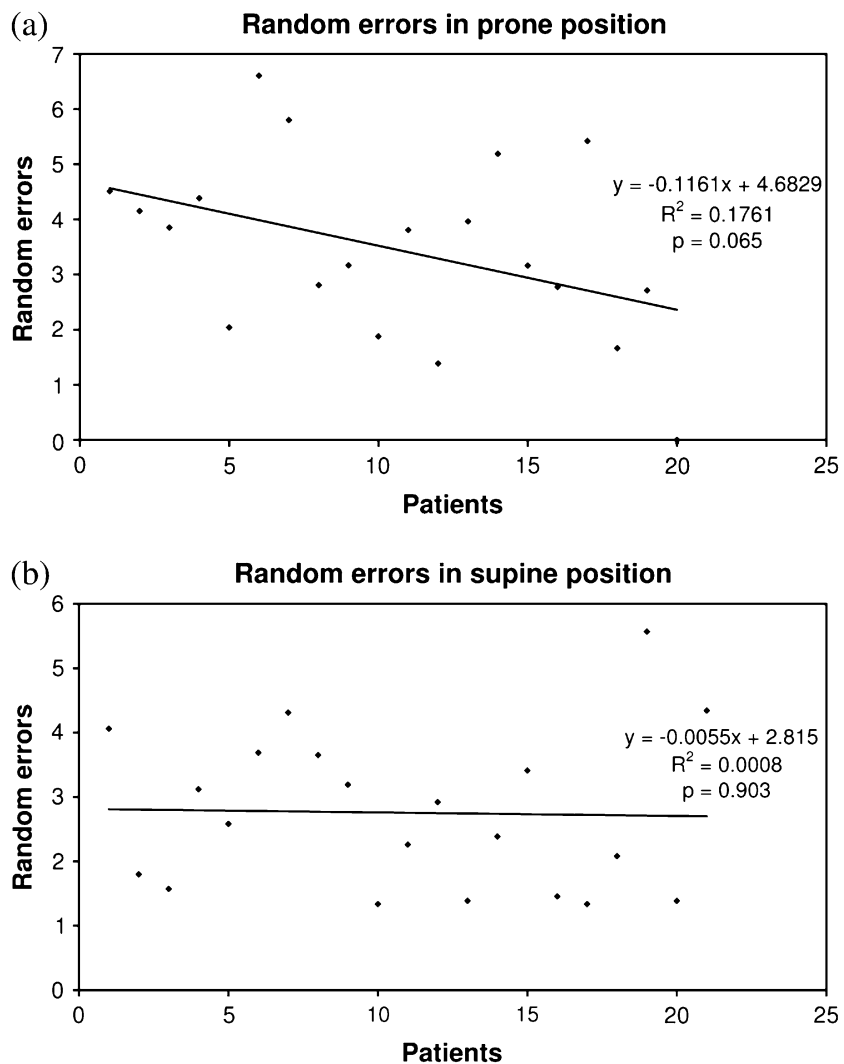

Fig. 2. Random errors for repositioning among the patients who received radiotherapy prone (a) and those received radiotherapy supine (b) by sequence of enrolment in the study. investigation of 35 patients with large pendulous breasts, Mahe et al. (10) found that when conventional tangents were used, the dose was $105 \%-110 \%$ in one third of patients. Despite the use of in-field segments, we observed hot spots at the top and bottom of the target breast in the prone position, which is consistent with the experience of Mahe et al. The application of intensity-modulated beams in our study may have played a role in the apparent lack of a relation between dose uniformity and breast size.

Because of the different shape of the chest wall when the patient is positioned prone, the lung volume included in the tangent fields is considerably less. All authors agree that lung doses are dramatically reduced if breast radiotherapy is performed with the patient prone (5-9). The beneficial effect of prone positioning on the protection of the ipsilateral lung is further enhanced if the almost absent intrafractional motion of the chest wall is taken into account for the calculation of safety margins around the CTV $(20,24,25)$.

When left-sided irradiation is performed, the irradiated volume of the heart is not reduced, despite the fact that less intrathoracic volume is exposed to radiation in the prone than in the supine position. Reports on heart doses are not concordant, however. Some studies suggest a reduction in heart doses as a result of prone positioning but do not provide direct comparisons with supine positioning $(5,8)$. Others are consistent with our results in showing no significant difference in doses to the heart as a function of the treatment position $(6,7,9)$. This finding may be accepted if the change in position of the heart by treatment position is taken into consideration. In fact, the prone position causes an anterior displacement of the heart within the thorax by $19 \mathrm{~mm}$ on average, as demonstrated by CT and MRI measurements in breast cancer patients receiving radiotherapy (26).

Because breast radiotherapy increases the risk of late contralateral breast cancer by $18 \%-34 \%$, special attention is necessary to protect the opposite breast $(3,4)$. Although some studies allude to the radiation dose to the opposite breast in the prone position, detailed dose-volume histogram data have not been provided $(5,6)$. No widely accepted dose constraints exist for the contralateral breast. We registered $V_{5 G y}$ and the mean dose to the healthy breast. In the first phase of the study, we detected higher doses to the opposite breast in the prone than supine position, a consequence of suboptimal positioning in the prone state. Following revision of the positioning method, no difference was observed in the second phase of the study. We consider careful application of the polyfoam wedge in the prone position, and of mask fixation in the supine position, to be important in removing the opposite breast from the radiation fields.

The largest prospective Phase I-II study on prone breast irradiation is that of Formenti et al. (8). Accelerated whole breast radiotherapy was feasible in 90 patients, with high setup reproducibility, although numerical data were not provided. In another feasibility study (10), prolonged adequate immobilization could not be achieved in 3 of 35 patients with large pendulous breasts in the prone position. In one retrospective study (11), $5 \%$ of the patients during prone breast 
radiotherapy complained of chest wall or rib pain, and 2 of 248 patients suffered a rib fracture (11), as did 1 of 35 in the previous study (10). All our patients considered the prone radiotherapy convenient and completed the course of radiotherapy. We believe that the comfortable positioning system was essential to achieve such good adherence to the protocol. It is our view that repositioning accuracy is a key condition for radiotherapy, particularly if inverse or forward intensity modulation is applied $(24,25)$. During simulation in 308 patients with various cancer sites, Schüller et al. (27) found that the repositioning accuracy was improved in the entire patient population if positioning aids or mask fixation were used, but this was not affected by prone or supine positioning. Breast irradiation was performed without mask fixation in the supine position for 64 patients. Of the various tumor sites, the breast exhibited the poorest repositioning accuracy. Displacement was carried out in 27 patients (42.2\%) and exceeded $1 \mathrm{~cm}$ in many cases. In another study of 25 breast cancer patients irradiated in the supine position (28), the isocenter displacement on simulation was $5.7 \mathrm{~mm}$ on average. Morrow et al. (20) studied interfractional error in repositioning in 15 patients and recommended image guidance during prone breast radiotherapy because of the need for frequent and large displacements. In agreement with our results, they observed no relation between the breast size and the repositioning accuracy. Interestingly, however, we found that the repositioning accuracy in the supine position is significantly worse in obese patients. To the best of our knowledge, no such data have been published previously. If confirmed, they indicate that increased attention must be paid to the position of overweight patients during breast radiotherapy. We believe that the relatively good repositioning accuracy in our study, was related to the comfortable positioning device used for both the prone and the supine position and to the mask fixation used in the supine position. Repositioning accuracy in the prone position improved over time, indicating the need for experience and expertise. Furthermore, our study warrants the development of mask fixation in the prone position, which would reduce setup uncertainty.

In other publications $(10,11)$, acute skin reactions after breast radiotherapy in the prone position were reported in similar incidences as among our patients. Mahe et al. (10) found that acute skin reactions were most frequent at the top and the bottom of the fields, in accordance with the high dose regions. In our study, radiodermatitis in the prone position was not related to the size of the breast or the dose inhomogeneity in it.

Merchant and McCormick (5) recommend breast radiotherapy in the prone position if the supine position is likely to result in unacceptable dose inhomogeneity or significant doses to normal tissues. We hoped to identify those patients who would benefit most from the prone position during breast radiotherapy. Because we could not detect any advantage of prone radiotherapy other than the absence of radiation exposure of the lung, we set out to identify those patient-related parameters that are associated with a higher lung dose if the patient is irradiated in a supine position. Consideration of breast volume, breast separation, and CLD as measures of the PTV shape indicated that only CLD was related to the dose to the ipsilateral lung. Thus, we recommend monitoring of the CLD as a primary measure for the indication of prone radiotherapy. Moreover, because the risk of early and late lung sequelae is strongly related to patient age (13), the presence of lung disease, and possibly to certain systemic therapies, these factors should be taken into account when a decision is made concerning the position used during breast radiotherapy.

\section{REFERENCES}

1. Kwa SLS, Lebesque JV, Theuws JC, et al. Radiation pneumonitis as a function of mean lung dose: An analysis of pooled data of 540 patients. Int J Radiat Oncol Biol Phys 1998;42: $1-9$.

2. Darby S, McGale P, Taylor CW, Peto R. Long-term mortality from heart disease and lung cancer after radiotherapy for early breast cancer: Prospective cohort study of about 300000 women in US SEER cancer registries. Lancet Oncol 2005;6: 557-565.

3. Early Breast Cancer Trialists' Collaborative Group (EBCTCG). Effects of radiotherapy and of differences in the extent of surgery for early breast cancer on local recurrence and 15-year survival: An overview of the randomised trials. Lancet 2005;366: 2087-2106.

4. Levi F, Randimbison L, Te VC, La Vecchia C. Cancer risk after radiotherapy for breast cancer. Br J Cancer 2006;95:390-392.

5. Merchant TE, McCormick B. Prone position breast irradiation. Int J Radiat Oncol Biol Phys 1994;30:197-203.

6. Griem KL, Fetherston P, Kuznetsova M, et al. Three-dimensional photon dosimetry: A comparison of treatment of the intact breast in the supine and prone position. Int $J$ Radiation Oncology Biol Phys 2003;57:891-899.

7. Kurtman C, Andrieu MN, Hicsönmez A, Celebioglu B. Threedimensional conformal breast irradiation in the prone position. Brazilian J Med Biol Res 2003;36:1441-1446.
8. Formenti SC, Gidea-Addeo D, Goldberg JD, et al. Phase I-II trial of prone accelerated intensity modulated radiation therapy to the breast to optimally spare normal tissue. J Clin Oncol 2007;25:2236-2242.

9. Buijsen J, Jager JJ, Bovendeerd J, et al. Prone breast irradiation for pendulous breasts. Radiother Oncol 2007;82:337-340.

10. Mahe M-A, Classe J-M, Dravet F, et al. Preliminary results for prone-position breast irradiation. Int J Radiation Oncology Biol Phys 2002;52:156-160.

11. Stegman LD, Beal KP, Hunt MA, et al. Long-term clinical outcomes of whole-breast irradiation delivered in the prone position. Int J Radiation Oncology Biol Phys 2007;68:73-81.

12. Grann A, McCormick B, Chabner ES, et al. Prone breast radiotherapy in early-stage breast cancer: A preliminary analysis. Int J Radiation Oncology Biol Phys 2000;47:319-325.

13. Kahán Z, Csenki M, Varga Z, et al. The risk of early and late lung sequelae after conformal radiotherapy in breast cancer patients. Int J Radiat Oncol Biol Phys 2007;68:673-681.

14. Fein DA, McGee KP, Schultheiss TE, et al. Intra- and interfractional reproducibility of tangential breast fields: A prospective on-line portal imaging study. Int $J$ Radiation Oncology Biol Phys 1996;34:733-740.

15. Lirette A, Pouliot J, Aubin M, Larochelle M. The role of electronic portal imaging in tangential breast irradiation: A prospective study. Radiother Oncol 1995;37:241-245. 
16. Hurkmans CW, Remeijer P, Lebesque JV, Mijnheer B. Set-up verification using portal imaging; review of current clinical practice. Radiother Oncol 2001;58:105-120.

17. Stroom JC, Heijmen BJM. Geometrical uncertainties, radiotherapy planning margins, and the ICRU-62 report. Radiother Oncol 2002;64:75-83.

18. Goodman KA, Hong L, Wagman R, et al. Dosimetric analysis of a simplified intensity modulation technique for prone breast radiotherapy. Int J Radiation Oncology Biol Phys 2004;60: 95-102.

19. Algan Ö, Fowble B, McNeeley S, Fein D. Use of the prone position in radiation treatment for women with early stage breast cancer. Int J Radiation Oncology Biol Phys 1998;40:11371140 .

20. Morrow NV, Stepaniak C, White J, et al. Intra- and interfractional variations for prone breast irradiation: An indication for image-guided radiotherapy. Int J Radiation Oncology Biol Phys 2007;69:910-917.

21. Moody AM, Mayles WP, Bliss JM, et al. The influence of breast size on late radiation effects and association with radiotherapy dose inhomogeneity. Int J Radiation Oncology Biol Phys 1994;33:106-112.
22. Johansen J, Overgaard J, Rose C, et al. Cosmetic outcome and breast morbidity in breast-conserving treatment. Results from the Danish DBCG-82TM national randomized trial in breast cancer. Acta Oncol 2002;41:369-380.

23. International Commission on Radiation Units and Measurements. Prescribing, recording and reporting photon beam therapy (supplement to ICRU Report 50) (ICRU Report 62). Bethesda, MD: ICRU Publications; 1999.

24. van Herk M. Errors and margins in radiotherapy. Semin Radiat Oncol 2004;14:52-64.

25. Saliou MG, Giraud P, Simon L, et al. Radiotherapy for breast cancer: Respiratory and set-up uncertainties. Cancer Radiother 2005;9:414-421.

26. Chino JP, Marks LB. Prone positioning causes the heart to be displaced anteriorly within the thorax: Implications for breast cancer treatment. Int J Radiation Oncology Biol Phys 2008; 70:916-920.

27. Schüller P, Bruns F, Hesselmann S, et al. Simulator verification of the accuracy of patient repositioning after virtual simulation. Strahlenther Onkol 2002;178:715-721.

28. Horst E, Schuck A, Moustakis C, et al. CT simulation in nodal positive breast cancer. Strahlenther Onkol 2001;177:511-516. 